\title{
Comunicación y desarrollo en las agendas internacionales ${ }^{1}$
}

\section{Communication and development in international agendas}

\author{
Federico Larsen ${ }^{2}$
}

Resumen: Las teorías sobre desarrollo y la comunicación social comenzaron a entrecruzarse en la segunda mitad del siglo XX, configurando luego el campo de la Comunicación para el Desarrollo. Las dos perspectivas principales de ambas disciplinas hasta los años 1980 -las teorías de la modernización y de la dependencia- protagonizaron también la disputa sobre la regulación del flujo informativo en los organismos internacionales. Más recientemente, los Objetivos del Milenio y la Agenda 2030 para el Desarrollo Sostenible retomaron la visión instrumental de la comunicación consolidada en los años 1990, aunque abierta al nuevo enfoque de la Comunicación para el Cambio Social. Este trabajo recorre los hitos de conformación del campo y propone algunas claves de lectura de su situación actual.

Palabras clave: Comunicación para el desarrollo, Comunicación para el cambio social, Desarrollo, Nuevo Orden Internacional de la Información.

Abstract: Development and social communication theories began to intersect in the second half of the 20th century, then shaping the field of Communication for Development. The two main perspectives of both disciplines up to the 1980s -the theories of modernization and the theories of dependence- also led the dispute on the regulation of the flow of information in international organizations. More recently, the Millennium Goals and the 2030 Agenda for Sustainable Development took up the instrumental vision of communication consolidated in the 1990s, though open to the new approach of Communication for Social Change. This work covers the milestones of conformation of the field, and proposes some reading guidelines for its current situation.

Key-words: Communication for Development, Communication for Social Change, Development, New World Information and Communication Order

DOI: https://doi.org/10.24215/23142766e060

\footnotetext{
${ }^{1}$ Recibido: 25/04/19. Aceptado: 26/06/2019

2 Periodista y docente. Licenciado en comunicación social con orientación en periodismo (FPyCS-UNLP); maestrando del Instituto de Relaciones Internacionales (IRI-UNLP). Mail: larsenfede@gmail.com
} 


\section{Introducción}

El binomio comunicación-desarrollo se ha consolidado como objeto de investigación a la par de su auge en la praxis de los agentes de la cooperación internacional. De allí que las acepciones y consideraciones acerca de ambos términos hayan ido modificándose en función de los intereses, concepciones e intenciones de quienes dispusieran de ellos. Es por eso que, con el objetivo de encarar este trabajo, daremos sólo algunas definiciones básicas para que se vayan enriqueciendo con los conceptos y avances teóricos elaborados en el desarrollo del campo, tal como sucedió en los últimos 60 años.

Entenderemos aquí a la comunicación como una dimensión de la realidad cuya finalidad es la de construir sentidos socialmente reconocibles. Como veremos, existen diferentes formas de concebir esa construcción y esos sentidos, pero podemos adelantar que nos limitaremos aquí al análisis de la significación social, dejando de lado la dimensión vincular de la comunicación ${ }^{3}$. El desarrollo será entendido, entinces, como una modalidad de intervención que busca modificar aspectos de la realidad en función de un orden social ideal que subyace y determina esa intervención (Cimadevilla 2008:102).

A partir de estos dos conceptos se intentará trazar una historización crítica de la relación entre comunicación y desarrollo, en los planos de la praxis y de la epistemología, hasta su consolidación como campo ${ }^{4}$ multidisciplinario que involucra tanto la comunicación como las teorías del desarrollo y las relaciones internacionales. Es decir, nos proponemos aquí bosquejar una respuesta a la pregunta: ¿de qué estamos hablando cuando nos referimos a comunicación para el desarrollo? Veremos luego cómo estos conceptos han tenido cierto protagonismo en los debates y las políticas de las organizaciones internacionales y cuál es el rol que tiene hoy en la agenda global de la cooperación al desarrollo.

Cabe destacar que este trabajo sólo pretende ser una aproximación al estudio de la comunicación para el desarrollo y no un compendio exhaustivo del este. Para profundizar conceptos o reparar omisiones, nos remitimos a la bibliografía esencial que se incluye en este texto.

\section{El desarrollo como modernización, la comunicación como difusión}

Cuando en 1958 el sociólogo David Lerner publicó para el Instituto Tecnológico de Massachusets The Passing of Traditional Society: Modernizing the Middle East, muchas de las teorías que se habían elaborado en los años precedentes acerca de qué es el desarrollo y cómo la comunicación podía aportar a ese proceso tomaron un cuerpo orgánico y una demostración científica. Lerner había dado el puntapié para la elaboración de una serie de teorías en el ámbito de la comunicación para el desarrollo que llamaremos Teorías de la Modernización. Su marco de elaboración es el periodo que le sigue a la Segunda Guerra Mundial y el comienzo de la Guerra Fría, marcado por el reacomodamiento de las zonas de

3 Cabe aclarar que la diferenciación entre comunicación vincular y social puede ser entendida como artificial o engañosa por diferentes autores. Véase al respecto Veron E. (1993), La semiosis social: fragmentos de una teoría de la discursividad. Barcelona: Gedisa o Barbero (1987).

4 Entendemos aquí el concepto de campo según la concepción de Pierre Bourdieu, quien lo define como una esfera de la vida social que se diferencia de las otras a partir de normas, creencia, habitus y una lucha hegemónica por el poder, las cuales dan sus distintas expresiones del capital interno al campo. 
influencia de los dos principales bloques del bipolarismo de la segunda parte del siglo XX. Las potencias estaban urgidas por la necesidad de expandir su esfera de dominación ya no sólo a través de la conquista y la guerra, sino que la cooperación internacional se había convertido en una nueva arma para seducir o someter a los países de esa parte del mundo que el presidente Harry Truman había bautizado como subdesarrollado en 1949. Este proceso neocolonial se desarrolló en el mismo periodo en el cual se registraba el lento proceso de descolonización, que permitió a los movimientos de liberación nacional de África y Asia el acceso a la independencia y al control de nuevos Estados, cuyo sostenimiento requeriría de recursos y conocimientos que los países centrales estaban más que dispuestos a ofrecer para expandir sus fronteras de influencia.

Para ordenar este proceso de desarrollo de los países pobres, nuevos o en vías de industrialización, desde las universidades y agencias estatales del norte del mundo se estableció una doctrina según la cual el objetivo debía ser la eliminación de las diferencias que existían entre los países pobres y los ricos. Esta óptica, marcada por una visión economicista del desarrollo, presuponía elevar los ingresos de las familias rurales de los países del sur a través de planes de industrialización, tecnologización y formación para que éstos pudieran parecerse más a los del norte. El desarrollo, desde esta perspectiva, consistía entonces en el acceso a tecnologías, saberes y consumo por parte de quienes no los poseían. A esta forma de entender el desarrollo le subyace la idea de que se trata de un proceso lineal que va desde la falta hacia el acceso, y donde en la meta a la que ambicionan los países no desarrollados se encuentran los países que sí han recorrido la escalera al desarrollo. Una obra clásica en este sentido es Las etapas del crecimiento económico: un manifiesto no comunista de W. W. Rostow, que, desde una perspectiva económica, supone la existencia de cinco etapas del desarrollo (sociedad tradicional, transición, despegue, madurez y consumo de masa), dentro de un sistema de formación de periodos históricos que comienza con un "impulso inicial hacia un crecimiento autosostenido", y basado en los saltos sucesivos que dan hacia adelante los "sectores principales" de la economía (Baran y Hosbawm, 1963:285). Para eso, se entendía como necesaria la inyección desde afuera de capitales que favoreciera ese salto y el comienzo del proceso evolutivo "natural" de la sociedad.

Ahora bien, Lerner y los sociólogos de la comunicación de la escuela que llamaremos difusionista se preguntaban cómo la comunicación podía facilitar y hasta acelerar el proceso de desarrollo en los países más pobres. Su teorización fue bastante simple: si el subdesarrollo es "no tener", entonces desde el punto de vista de la comunicación lo que falta es información. Según esta perspectiva, entonces, la pobreza es causada por falta de información y conocimiento relacionado a cómo salir de ella, y lo que sí existe (las culturas tradicionales) se convierte en una barrera al desarrollo. Los países que aún no han llegado al desarrollo que ostentan Europa y EE.UU. se encuentran entonces en una fase atrasada en su camino hacia la civilización y, para alcanzarla, se deben superar distintas etapas sucesivas, desde las sociedades de tipo tradicional hasta la modernidad. Pero la persistencia de rasgos tradicionales en sus culturas es un síntoma de atraso que puede ser modificado y los medios de comunicación de masas pueden ser un agente para la modernización ${ }^{5}$.

5 Un análisis pormenorizado se encuentra en otro de los principales exponentes del difusionismo, véase Schramm W. (1964) Mass Media and National Development: The role of Information in the Developing Countries, San Francisco: Stanford University Press 
Esas son justamente las conclusiones a las que llegó el estudio de Lerner. Los investigadores realizaron una serie de encuestas para medir las opiniones de ciudadanos de diferentes países de Oriente Medio acerca de las transmisiones de tres medios de comunicación extranjeros: la BBC inglesa, Radio Moscú y la estadounidense estatal Voice of America. En sus consideraciones, los sociólogos de la comunicación aseguran que, en el pasaje del "Estado tradicional" al "Estado moderno", es condición indispensable la difusión de una "actitud psicológica de movilidad", una disposición al desarrollo, y que ésta es efectivamente multiplicable a través de los medios de comunicación masivos ${ }^{6}$ (Mattelart, 1997:36).

El rol de la comunicación en el debate sobre el desarrollo es, entonces, el de difundir las innovaciones elaboradas en el norte, lugar/concepto que detiene el privilegio exclusivo del saber, y de esta manera transferir el conocimiento hacia el sur. Este proceso, que en algunos países de América Latina fue adoptado de muy buena gana, coincidió con dos grandes cambios en la producción tecnológica: por un lado, la llamada "Revolución Verde" propiciada por la reconversión de la industria bélica en industria agrícola y que inundó de nuevas tecnologías al sector primario de los países más pobres, especialmente los de América Latina, más expuestos al ejemplo revolucionario cubano; y, por el otro, la gran aceleración de la producción tecnológica para la comunicación y sus variantes en la difusión de los nuevos medios ${ }^{7}$. El método para la difusión del conocimiento, motor del desarrollo, es entonces el del uso intensivo de campañas a través de los medios masivos de comunicación. Se delinea allí un esquema vertical de comunicación basado en la intención del donante, que a partir de un diagnóstico generalizado define una estrategia de mercadeo en un espacio puntual, cuyo objetivo principal es el cambio en un comportamiento, la erradicación de las formas tradicionales de pensamiento. Este modelo, que en comunicación se conoció en los años 1930 como el de la "Aguja Hipodérmica", está aún hoy en las bases de las estrategias de comunicación de muchas agencias de cooperación (McAnany, 2010:8), las que difunden mensajes propagandísticos sobre la salud, el cuidado del medio ambiente o la educación, y cuya principal evaluación es cuantitativa, como por ejemplo a partir del número de reproducciones en las redes o en los medios masivos de comunicación de un determinado mensaje.

El paradigma modernista del desarrollo equivale al difusionismo en comunicación, cuyo principal objetivo es la modificación de una conducta para que pueda ser asumido el camino hacia el desarrollo. Pero esta perspectiva ignoraba por completo las necesidades específicas de las comunidades en las que pretendía operar, estaba claramente cargado de

6 El estudio de Lerner se realizó junto con el Boureau of Applied Social Research de Felix Lazarsfeld, eminencia en los estudios de la sociología funcionalista de la comunicación que había logrado fundar su Boureau en la universidad de Columbia con la financiación del Ministerio de Guerra de los EEUU, con el fin de aplicar los estudios de la Propaganda-War comenzados hacia finales de la II Guerra Mundial por su principal mentor, Harold Lasswell. Véase al respecto Lazarsfeld P. F. (1953), "The prognosis of international communication research", Oxford, Public Opinion Quarterly, vol. 16, pp. 481-490.

7 Roncagliolo (1989) inclusive individua dos variables en la difusión de la radio-televisión en los países en desarrollo tras la II Guerra Mundial: una variable europea, donde los nuevos medios son concebidos como un servicio público con una finalidad educativo-difusionista, y que fue la más utilizada en Asia y África; y una variable norteamericana, donde la radiodifusión se ejerce en función de una concepción privado-comercialpublicitario, y que ha estado a la base de los proyectos de radiodifusión en América Latina. Esto, según el autor, aún hoy marca la diferencia en el desarrollo del campo de la televisión en el mundo. 
prejuicios darwinistas etnocéntricos y una perspectiva ahistórica, que generó fuertes resistencias en los países del sur del mundo.

\section{Las teorías de la dependencia y el paradigma participativo}

En los años 70 el modelo economicista de explicación de las diferencias entre los países entró en crisis. Según Rostow, el problema del desarrollo era interno, es decir que los mismos países subdesarrollados cargaban con la responsabilidad de no haber avanzado en su proceso histórico. A esta visión se le contrapuso un pensamiento que tomaba como centro del análisis al sistema capitalista internacional, y no los Estados o comunidades. Éste podía ser explicado a partir de dos lógicas principales: la lógica del dominio, elaborada por las corrientes marxistas, según la cual la idea misma del desarrollo no es otra cosa que una elucubración del imperialismo para imponer su modelo, el capitalismo mundial, dentro del cual no hay desarrollo posible; y la lógica de la dependencia, según la cual desarrollo y subdesarrollo son dos caras de una misma moneda, y que pone el acento en la desigualdad sistémica entre un centro desarrollado y una periferia del mundo que no lo es. El diálogo permanente entre estas dos lógicas fue el espacio de elaboración de un conjunto de teorías que llamamos genéricamente Teorías de la Dependencia. Según estos enfoques, el subdesarrollo no es una etapa previa al desarrollo, sino una condición necesaria para la supervivencia del sistema capitalista mundial. La relación centro-periferia no es un problema interno de los países subdesarrollados, los cuales no consiguen llegar al nivel de los desarrollados, sino de relaciones de poder internacionales. La periferia depende del sistema-mundo capitalista que determina sus estructuras económicas, sociales y políticas, en función de los intereses del centro del sistema ${ }^{8}$.

Este enfoque, retomado por infinidad de autores, tuvo, en el estudio de la comunicación, un primer desarrollo en el centro de la producción capitalista mundial que impactó luego con fuerza en su periferia. Es el caso de los trabajos de Herbert Schiller, que en 1969 publicó un libro crucial para el estudio de la Dominación/Dependencia en los medios de comunicación y la cultura: Comunicación de masas e imperialismo yanqui. Schiller elaboró el concepto de imperialismo cultural a partir del análisis del rol del complejo militar-industrial estadocunidense en la industria cultural e informativa. Según él, en el mundo surgido tras la Segunda Guerra Mundial, el poder militar ya no era suficiente para garantizar la primacía estadounidense a nivel internacional. Era necesario explorar un nuevo campo de dominación que estaba logrando interesantes y agigantados avances científico-tecnológicos: la comunicación social.

Los medios masivos de comunicación se convierten así en una herramienta fundamental para la dominación semántica de sociedades muy diferentes de la que eran sede de emisión de los mensajes. La particularidad de la teoría de Schiller es que introduce la voluntad de los sectores dirigentes de las sociedades bajo dominación de seguir los preceptos impuestos por los dominantes. Schiller define, entonces, el imperialismo cultural como:

8 Obras fundantes de esta perspectiva son Cardoso y Faletto (1969), Dependencia y desarrollo en América Latina, Buenos Aires:Siglo XXI; Gunder Frank (1965), Capitalismo y subdesarrollo en América Latina, México:Siglo XXI ; Wallerstein (1974) El moderno sistema mundial, México:Siglo XXI; entre otros 
El conjunto de procesos por los que una sociedad es introducida en el seno del sistema moderno mundial y la manera en que su capa dirigente es llevada, por la fascinación, la presión, la fuerza o la corrupción, a moldear las instituciones sociales para que correspondan con los valores y estructuras del centro dominante del sistema o para hacerse su promotor [...] Existe un poderoso sistema de comunicaciones para asegurar no una sumisión sufrida de mala gana, sino una alianza con los brazos abiertos en las áreas penetradas, identificando la presencia norteamericana con la libertad: libertad de comercio, libertad de palabra y libertad de empresa (Schiller, 1976:13).

En los EE.UU. entre los años 60 y 70 se elaborarán decenas de estudios que rompen con la idea que la sociología funcionalista había elaborado de la comunicación, según la cual los medios eran un instrumento para el mantenimiento del orden democrático, cuyo rol de "ordenadores de la sociedad" aseguraba la prosecución de la paz y la armonía de la sociedad, y la transmisión de los más altos valores de la humanidad y la cultura ${ }^{9}$. El surgimiento de las perspectivas críticas sobre los medios puso en tela de juicio esta idea e inauguró una discrepancia que el semiólogo italiano Umberto Eco resumió en el título de su obra de 1964: Apocalípticos e Integrados. A quienes entienden los medios de comunicación como un instrumento de la sociedad de masas para integrar cada vez más personas en los beneficios de la democracia occidental, se le oponen aquellos que entienden la comunicación como un instrumento de poder en el marco de un sistema internacional desigual y opresor. Los medios, lejos de considerarse como instrumentos de la democracia, son herramientas de dominación en manos de grandes potencias que los usan para la defensa de sus intereses.

Este último enfoque encontró en América Latina un importante centro de elaboración y estudio, coadyuvado por la formulación del pensamiento periférico continental en las relaciones internacionales. Como indica Beltrán (2006:56), en América Latina "primero fue la praxis". Es esta una de las características más destacadas de la construcción teórica en comunicación en el continente, su permanente y virtuosa relación con proyectos educativos y comunicacionales inspirados por movimientos de base campesinos, indígenas, obreros y feministas, entre otros.

Luego, una enorme cantidad de autores latinoamericanos (Antonio Pascuali, Juan Díaz Bordenave, Mario Kaplún, Rafael Roncagliolo y Fernando Reyes Matta, para citar sólo algunos ejemplos), inspirados en las experiencias de las radios mineras bolivianas o las radioescuelas de los curas tercermundistas en Colombia, comenzó a elaborar un modelo alternativo al de las teorías de la modernización, que dieron vida al paradigma participativo de la comunicación para el desarrollo ${ }^{10}$. Para los estudiosos de la comunicación del continente no se podía seguir endilgando a la falta de información la culpa del subdesarrollo, sino que sus causas debían ser buscadas en las condiciones estructurales del sistema capitalista. Es decir,

9 Esta perspectiva se verá reflejada en los años 90 en expresiones tales como "aldea global", "sociedad de la información" o "autopistas de la comunicación", que niegan el escenario de conflicto social permanente.

10 No se puede soslayar de todas maneras el inmenso aporte que han dado las experiencias prácticas de movimientos sociales y comunidades de Asia y África. Son claro testimonio de ello las recopilaciones de Gumucio Dargon (2001). Si bien se tiende a definir a América Latina como el entorno de gestación de la comunicación participativa para el desarrollo, por la cantidad de experiencias prácticas y la emergencia de un núcleo de pensadores críticos que compartieron los mismos debates. 
el campesino latinoamericano no es pobre por no conocer las tecnologías que producen los EE.UU. o Europa, sino por las condiciones estructurales de la dependencia del continente frente a los centros de poder, interesados en imponer no sólo su tecnología sino también su forma de usarla, su lógica de acceso y, en definitiva, su cultura ${ }^{11}$.

Se partía de la idea de que el modelo vertical del difusionismo, lejos de generar las condiciones para el desarrollo, profundiza las condiciones de la dependencia. Por eso, el cuestionamiento se centró en tres aspectos del modelo: el concepto de comunicación, los métodos propuestos para la intervención y el objetivo del proceso. La concepción de comunicación que se proponía era la del intercambio de saberes a través del diálogo y del reconocimiento mutuo. Este concepto niega la posibilidad de una transferencia en sentido vertical del saber, en una concepción bancaria ${ }^{12}$ que pretende llenar de conocimiento a aquél que de él es huérfano. Por el contrario, la comunicación es imposible sin la interacción y la participación. En ella los sujetos son participantes activos y conscientes de la construcción de su propio devenir; de allí que muchos autores comiencen a partir de aquí una larga discusión en torno a la diferencia entre información y comunicación ${ }^{13}$ : la primera es una difusión universal y estándar de un determinado mensaje a un público atomizado e inerte; mientras que la segunda necesita de un modelo dialógico, donde el receptor es participante activo y con capacidad de intervención sobre el proceso que no puede ser universal e igual para todos sino lo más pormenorizado posible y respetuoso de las culturas, los valores y las tradiciones locales, a partir de los cuales los mismos participantes determinarán el método de intervención. El valor estará entonces puesto en las necesidades de las comunidades y no en la prioridad de los donantes de la cooperación. El tercer cuestionamiento tiene que ver con el objetivo del proceso. Las teorías de la dependencia introducen un concepto que será retomado más adelante y que tiene que ver con la idea de Comunicación para el Cambio Social. La meta que se propone para los procesos de comunicación en el ámbito de la cooperación internacional no es ya el cambio de determinados comportamientos ligados a lo tradicional, sino la generación de procesos a largo plazo de cambio en las condiciones estructurales que determinan el desarrollo.

Se perfilan así dos modelos que fundan el campo de la comunicación para el desarroIlo: un modelo vertical y jerárquico, basado sobre la universalidad de los mensajes y la masividad de la intervención, y un modelo horizontal y participativo, basado en la particularidad y comunión del diálogo.

11 En el ámbito latinoamericano, uno de los trabajos señeros de la teoría del imperialismo cultural es Dorfman A. y Mattelart A. (1972), Para leer al Pato Donald. Comunicación de masa y colonialismo, Buenos Aires: Siglo XXI, en el cual los autores demuestran la intencionalidad que se esconde detrás de las historietas de Walt Disney se esconde una forma de entender la realidad social y las relaciones de poder y de género al cual el público latinoamericano está permanentemente expuesto.

12 El aporte de Paulo Freire y su Pedagogía del Oprimido fue fundamental en la elaboración de la base conceptual de la Comunicación para el Desarrollo. Especialmente su idea de concientización, diametralmente opuesta a la de difusión, en la cual la realidad es conocida a partir de la toma de conciencia por parte del oprimido de su condición, y de allí elaborar una aproximación crítica a un problema o fenómeno social. Véase Freire P. (1973), ¿Extención o comunicación?: La concientización en el medio rural, Mexico:Siglo XXI

13 Un trabajo reciente pero considerado ya entre los más influyentes acerca de esta cuestión es Wolton $D$. (2010), Informar no es comunicar, Barcelona:Gedisa 


\section{El debate internacional}

Las divergencias entre los modelos difusionista y participativo tuvieron su resonancia en la diplomacia internacional en los años 60 y 70 . Hasta ese momento, y desde el siglo XIX, los EE.UU. habían desarrollado una doctrina que se había convertido en su posición oficial a nivel internacional en torno a la comunicación, conocida como Free Flow of Information (Matelart, 1997:79). Según esta doctrina, la información y los mensajes debían circular libremente a lo largo y a lo ancho del planeta, y la restricción o reglamentación del flujo de información equivaldría a la imposición de formas de censura características de los Estados totalitarios. Los EE.UU. asumían, de esta manera, su rol de emisores en el modelo comunicacional de difusión del desarrollo y promovían la total apertura de los demás países, especialmente los del Sur, a sus mensajes y sus tecnologías. Las asimetrías económicas y políticas hacían que ese flujo fuera principalmente en un solo sentido. Así, la libre circulación de la información garantizaba claramente la hegemonía cultural, política, tecnológica y económica de occidente, a través de las iniciativas públicas de los países centrales o de sus empresas transnacionales (Castro Ruano, 1999:195). Pero las teorías de la dependencia y los paradigmas ligados a las posiciones más críticas en relaciones internacionales sostenían que el libre flujo de la información era una forma injerencista del imperio para socavar la soberanía de otros pueblos, y cada Estado tenía entonces la prerrogativa de defenderse ante semejante ataque a través de la regulación de los flujos informativos y del uso de las tecnologías occidentales. Estos planteamientos sirvieron claramente de justificación para varios países del bloque socialista y periféricos frente a la acusación de censura y manipulación de la información movidas en contra de sus gobiernos (Mattelart, 1997:82), pero también fueron el impulso político-ideológico para la elaboración de Proyectos Nacionales de Comunicación que regularan el flujo de mensajes y tecnologías, aunque eran insuficientes ante la asimetría de la estructura internacional. Para los países periféricos, era entonces necesario rediscutir esa estructura.

Desde 1968 en la UNESCO se había promovido un debate tendiente a la evaluación de los efectos de los medios de comunicación de masas en las sociedades modernas. Se instaló en ese momento el concepto de "comunicación en sentido único", que el Director General de la organización, el senegalés Amadou-Mahtar M'Bow, describió al concluir la cumbre de la UNESCO en Nairobi en 1976 del siguiente modo:

[L]a difusión de la información se realiza en gran medida en sentido único, a partir de algunos centros situados sobre todo en los países industrializados. Esta información refleja necesariamente las preocupaciones, las aspiraciones, es decir, el punto de vista, de las sociedades en donde tienen su origen, de tal modo que los organismos que constituyen su soporte tienden, con intención o sin ella, a instaurar una dominación de hecho, que puede imponer modelos culturales (Arenal 1985:16).

El mismo M'Bow propició en 1977 la creación de una comisión internacional de estudio de los problemas de la comunicación, presidida por el irlandés Sean MacBride, premio Lenin y Nobel por la Paz y fundador de Amnistía Internacional. El trabajo de la comisión desembocó, en 1980, en la publicación de un volumen titulado Un solo mundo, voces múltiples, conocido mundialmente como "Informe MacBride". La comisión, compuesta por especialistas en el ámbito de la comunicación y el periodismo provenientes de todas partes del 
mundo ${ }^{14}$, también retomó la idea de "información en sentido único", definida como "una corriente de noticias, datos, mensajes, programas y productos culturales, que va casi exclusivamente de los países grandes a los pequeños, de quienes tienen el poder y los medios tecnológicos a quienes están privados de ellos, de los países desarrollados a los países en desarrollo y, en el plano nacional, del centro del poder hacia abajo" (MacBride, 1980:252).

Estas definiciones retomaban claramente los documentos que ya habían sido elaborados en las cumbres del Movimiento de países No Alineados (MNOAL) en los años precedentes, y que culminaron con la V Conferencia de la Cumbre de los Países No Alineados, celebrada en Colombo en agosto de 1976, en la que aparece por primera vez una propuesta orgánica por parte del movimiento en el ámbito de la comunicación: el Nuevo Orden Internacional de la Información y la Comunicación (NOMIC). El análisis de la situación informativa internacional era claro. Las políticas difusionistas y la concentración de la producción tecnológica en los países desarrollados tenía una clara influencia sobre la imposición de modelos culturales en todo el planeta. Para los países del MNOAL, el establecimiento de un nuevo orden comunicacional era inseparable de la creación de un nuevo orden económico y viceversa. Por primera vez, en el ámbito de una organización internacional, se planteaba con fuerza una estrecha vinculación entre las políticas de comunicación y el desarrollo desigual del mundo, entendidos como problemas inseparables entre sí.

EI NOMIC proponía modificar por completo el sistema mediático internacional a partir de una serie de principios que se podrían resumir en: 1) la sustitución de un sistema de comunicación en sentido único por una circulación multidireccional y equilibrada de la información; 2) la descolonización del contenido de la información, considerando que sus condiciones de producción se establecen en los centros del poder; 3) la democratización de la comunicación, al ampliar el significado mismo de derecho a la información entendido como derecho a obtener, realizar y difundir información y consagrando la libertad y derecho de acceso a los medios disponibles para su distribución a toda la humanidad; 4) la redistribución transnacional de los recursos de la comunicación como frecuencias, satélites y bancos de datos, entre otros; 5 ) la promoción de sistemas nacionales de comunicación en los países periféricos; y 6) el establecimiento de mecanismos de ayuda financiera para el acceso soberano y definitivo a los sistemas de comunicación internacional (Arenal, 1985:19).

Estos seis principios se pueden encontrar en las conclusiones y 82 recomendaciones presentadas en el Informe MacBride. En sus conclusiones, el informe entiende que el derecho a buscar, recibir y difundir información es un derecho humano individual y colectivo, el que a la vez necesita de un nuevo orden internacional para su garantía. Al mismo tiempo exhorta a "otorgar mayor importancia a la eliminación de los desequilibrios y disparidades en la comunicación y sus estructuras, y particularmente en las corrientes de información", y define el establecimiento del NOMIC como un "proceso continuo de cambio en la naturaleza de las relaciones entre las naciones y dentro de ellas" (MacBride, 1980:245).

La presentación del informe ante el Director General de la UNESCO generó la férrea oposición de los países occidentales. Pero lo que precipitó el debate entorno a los flujos

14 Los 16 miembros que componían la Comisión, además del presidente, eran: Elie Abel (USA), Huben Bcuve-Mery (Francia), Elebe Ma Elie Ekonzo (Zaire), Gabriel García Márquez (Colombia). Sergei Losev (URSS), Mochtar Lubis (Indonesia), Mustapha Masmoudi (Túnez), Michio Nagai (Japón), Fred Isaac Akporuaro (Nigeria), Bogdan Osolnik (Yugoslavia), Gamal El Oteifi (Egipto), Johannes Pieter Pronk (Países Bajos), Juan Somavia (Chile), Boobli George Verghese (India) y Belly Zimmerman (Canadá). 
internacionales de la información fue la reacción del gobierno de Reagan ante la legitimidad que el contenido del informe MacBride estaba cosechando en todo el mundo. La delegación estadounidense en la UNESCO logró el Ilamado a la Conferencia Intergubernamental sobre la Cooperación en lo concerniente a las Actividades, Necesidades y Programas de Desarrollo de la Comunicación, celebrada en París en 1980, donde propuso la creación de un ente descentralizado para la creación de un fondo de financiación para el fomento de la comunicación en los países en vía de desarrollo, que en los planes de Washington quedaría bajo dominio estadounidense. Pero esa propuesta fracasó y, en cambio, se estableció la creación del Programa Internacional para el Desarrollo de la Comunicación (PIDC), bajo la órbita de la UNESCO, que aún hoy financia proyectos comunicacionales en los países periféricos.

Si bien la creación del PDIC había significado una clara moderación de los términos del debate planteado por el NOMIC -y su histórica escasez en la actividad de la comunicación para el desarrollo es otro síntoma de ello-, los países centrales insistieron en la necesidad de abandonar el proyecto. El aparente éxito de los preceptos del NOMIC los Ilevaría a estrategias más agresivas. Luego de una serie de acusaciones de ideologización y politización en sentido anti-norteamericano de la UNESCO -en buena parte debido al debate en torno al libre flujo de la información-, los EE.UU. abandonaron la organización en 1984, seguidos por Gran Bretaña y Singapur al año siguiente. La UNESCO perdía, de esta manera, capacidad de acción y legitimidad como foro de debate, y la discusión en torno al NOMIC, ya debilitada inclusive por diferencias internas entre los países periféricos y la pérdida de gravitación diplomática del MNOAL, desapareció de la agenda (Quirós y Sierra, 2016:50). El del NOMIC, junto al informe MacBride, fue hasta la fecha el proceso más importante de debate internacional sobre comunicación, y su resolución fue casi nula.

\section{La comunicación para el cambio social}

En los años 80 comienza lo que Beltrán llama la "Década perdida" de América Latina, marcada por un profundo reflujo en la elaboración teórica y la aplicación de los paradigmas de la comunicación para el desarrollo (Beltrán, 2006:66). Este déficit se debe a diferentes factores: por un lado, la depresión económica que acompañó el retorno de gobiernos democráticos en los países del sur global concentró las miradas de los investigadores y la academia nuevamente en el desarrollo de los factores comerciales y financieros de las economías. Por el otro, el fracaso de los debates entorno al NOMIC y la pérdida de gravitación diplomática de los países periféricos, llevaron al ocultamiento de las fricciones entre los actores internacionales de la comunicación.

En el ámbito de las teorías del desarrollo, los años 1980 vieron el triunfo de las concepciones que subrayaban la necesidad de un esfuerzo, muchas veces cargado de renuncias y políticas públicas "dolorosas", para lograr un mejoramiento en los países subdesarollados. Estos conceptos, que Sen (2001:68) llama BLAST -acrónimo en inglés de la expresión "sangre, sudor y lágrimas" -, encontraron en el paradigma neoclásico del ajuste de la estructura estatal una expresión universalizada por el nuevo orden unipolar de los 90 . El conflicto generado por las dicotomías en las conceptualización de la comunicación para el desarrollo de las décadas anteriores (horizontal/vertical, colectivo/individual, participación/difusión, concientización/persuasión, etc.) fue sustituido, en este nuevo clima de época, por los conceptos universalizadores de "aldea global" o "sociedad de la información". Esta concepción elimina todo indicio de la conflictividad intrínseca de cualquier forma de comunicación y pone 
el acento en la convergencia entre nuevas tecnologías de la información, capacidades sociales y necesidades económicas, que se unen en un círculo virtuoso para favorecer el desarroIlo de las sociedades. El acento se puso principalmente en la capacidad tecnológica de las sociedades para facilitar el flujo de información a nivel planetario, y se lleva a un segundo plano el debate en torno al contenido y las condiciones de producción de esos mensajes.

En el ámbito de la teoría de la comunicación social, muchos estudios pusieron el acento en la construcción de identidades de los estudios etnográficos y en el ámbito internacional, en lo que se llamó el "efecto CNN". A partir de esta perspectiva, los estudios en comunicación se concentraron en el rol que cumplen las imágenes transmitidas a nivel global por ese canal, tanto en la audiencia (que presionará a sus gobiernos en contra o a favor de los procesos retratados) como en los funcionarios responsables (que modificarán sus conductas para calmar o agitar la opinión pública) ${ }^{15}$.

Es decir, el estudio sobre la comunicación a nivel internacional se concentró en los años 90 sobre los efectos de la concentración mediática y el comienzo de la convergencia tecnológica a nivel de masas como motores de la generación de procesos de cambio en los países en desarrollo.

Sin embargo, a finales de la década, una iniciativa de la Fundación Rockefeller volvió a poner la atención en la noción de comunicación para el desarrollo e inauguró un tercer paradigma fundamental en el campo: la comunicación para el cambio social. El concepto fue elaborado tras las conferencias de Bellagio (1997) y Cape Town (1998), a partir de las cuales se definió como:

un proceso de diálogo privado y público, a través del cual las gentes deciden quiénes son, qué quieren y cómo pueden obtenerlo. Por cambio social entendemos un cambio en la vida de un grupo social, de acuerdo a los parámetros establecidos por ese mismo grupo. Esta aproximación busca especialmente mejorar las vidas de los grupos marginados (tanto política como económicamente), y está guiada por los principios de la tolerancia, auto-determinación, equidad, justicia social y participación activa de todos (Gray-Felder D. y Deane J. 1999:6).

Además de la teoría, la comunicación para el cambio social reivindica la práctica establecida durante décadas por parte de actores sociales entre los más diversos en el mundo. Radios comunitarias, pequeñas televisoras educativas, proyectos de cooperación financiados por multinacionales, toda experiencia que surgiera desde y para las propias comunidades para el fortalecimiento de los procesos comunicacionales hacia adentro y hacia afuera,

15 Estas perspectivas reflejan claramente la idea que la escuela funcionalista tenía de la sociedad. Su intención es analizar el nivel de influencia que los medios logran sobre las masas -y a través de ellas sobre el poder político-, teniendo como únicos elementos de estudio el emisor y el mensaje. Desde una visión positiva, defienden la idea de que los medios, en cuanto contrapeso del poder político, influyen para establecer un equilibrio en el cual el Estado, además de rendir cuentas por sus acciones, debe modificar sus actos en función de las reacciones de la opinión pública. Los medios cumplirían así una función importantísima en el sostenimiento de la democracia y sus periodistas y empresarios, junto con los funcionarios públicos, actuarían cuales contralores y vigilantes del orden social. Pero esta perspectiva va aún más allá, dando a los medios de comunicación no sólo el rol de guardianes sino que podrían modificar inclusive la agenda de política exterior de los países. Algunos autores llegaron a afirmar que las intervenciones estadounidenses en Somalia, Haiti y Bosnia en los años 90 se dieron justamente en función del "efecto CNN", y no por la persecución de intereses propios del Departamento de Estado. Demás está aclarar que no acordamos con esta idea. 
podía ser considerada una fuente de este paradigma. Gomucio Dagron (2001), en un trabajo ya célebre en este tipo de estudios, recopiló 50 experiencias que, a su entender, podían ser consideradas ejemplos de comunicación para el cambio social. De allí intentó trazar un perfil de lo que se entiende por comunicación participativa para el cambio social, a partir de su horizontalidad (los pueblos asumen el control de los procesos y los contenidos de la comunicación), su especificidad (el proceso tiene que ser pensado a partir de cada comunidad o grupo social y no ser una adaptación de formatos estándar) y su carácter comunitario (donde lo que cuentan son las necesidades de la comunidad, no las exigencias, los tiempos o los deseos de los donantes).

Se introducen aquí evidentemente conceptos propios del paradigma participativo pero decurtado de la perspectiva crítica y contrahegemónica que animaba a las teorías de la dependencia. Si bien la comunicación para el cambio social reconoce la persistencia de asimetrías políticas o económicas, evita poner el acento en los intereses, procesos e intenciones que generan esas injusticias para concentrarse en la participación de las comunidades; de allí que varias instituciones de diferente índole hayan querido profundizar en el concepto para poder aplicarlo en sus agendas de cooperación internacional. A partir de la primera década de los años 2000, se multiplicaron los documentos oficiales de organismos internacionales que exhortaban al estudio y aplicación de los procesos de comunicación para el cambio social (Unicef 2011, FAO 2007 y 2008, Banco Mundial 2007 y 2008, Tufte y Mefalopulos 2009 y Mefalopulos 2008).

El punto más importante de este proceso ha sido seguramente el Congreso Mundial de Comunicación para el Desarrollo (WCCD), convocado por el Banco Mundial, la $\mathrm{FAO}^{16} \mathrm{y}$ The Communication Initiative, en Roma en 2006. El Consenso de Roma ${ }^{17}$, fruto de esa conferencia, retomó en gran medida las guías que habían trazado las reuniones de Bellagio y Cape Town, al reivindicar la dimensión participativa de la comunicación para el desarrollo. Esta es definida como:

un proceso social basado en el diálogo usando un amplio abanico de instrumentos y de métodos. Se refiere también a la búsqueda del cambio a diferentes niveles que incluyen escuchar, construir confianza, compartir conocimiento y habilidades, desarrollar políticas, debatir y aprender para lograr cambios sostenibles y significativos. No se trata de relaciones públicas ni de comunicación corporativa (Unicef, 2011:1).

En sus recomendaciones, la WCCD instó a los Estados y las instituciones para la cooperación a que incluyan la comunicación "desde el comienzo" y "como elemento fundamental" de sus programas, a que expandan las inversiones en los ámbitos clave para la comunicación

16 Hay que remarcar que la FAO, dentro de los organismos internacionales en funcionamiento, ha siempre tenido una postura muy particular con respecto a la comunicación que la ha distinguido en varias ocasiones. Cabe destacar que la atención dada por la tecnología agropecuaria y la producción de los alimentos en el paradigma modernizador tuvieron justamente a esta organización como facilitador de buena parte de los programas de cooperación. A partir de ese rol privilegiado es que uno de sus principales dirigentes, Colin Fraser, publicó junto con su esposa, Sonia Restrepo (1998), Communicating for Development: human change for survival, considerada como una de las obras fundamentales del campo de la comunicación para el desarrollo desde la perspectiva de las instituciones internacionales.

17 Disponible en http://siteresources.worldbank.org/EXTDEVCOMMENG/Resources/RomeConsensus07.pdf [último acceso 06/04/2019] 
para el desarrollo, a que adopten políticas y leyes que la faciliten, a que incluyan indicadores que permitan el monitoreo y evaluación de los procesos de comunicación en los proyectos de cooperación, y a que fortalezcan las redes internacionales de investigación y difusión del enfoque de la comunicación para el desarrollo (FAO, 2007:21). El campo había encontrado, a partir de estas definiciones, un nuevo nombre institucionalizado, comunicación para el cambio social, pero la práctica estaba aún lejos de sus preceptos teóricos.

\section{La comunicación en la agenda actual del desarrollo}

Este proceso se da en el marco de una serie de modificaciones más generales en torno al concepto de desarrollo que traerán diferentes impactos en los órganos decisorios y las aplicaciones de los conceptos de comunicación para el desarrollo y comunicación para el cambio social en los albores del siglo XXI. Un primer factor para el análisis es el desplazamiento del foco de acción en el ámbito de las iniciativas de cooperación internacional desde las necesidades de una comunidad hacia las oportunidades que esta tiene en base a sus capacidades. Es decir, del enfoque economicista hegemónico en los años 80 y 90 se pasó a una visión más atenta a las personas, para las cuales el ingreso -principal variable de medición del desarrollo de los países hasta ese momento- sólo es un medio para poner en funcionamiento distintas capacidades. En 1990 el Programa de Naciones Unidas para el Desarrollo (PNUD) comenzó a utilizar su Indice de Desarrollo Humano para cuantificar los logros obtenidos en los distintos países en tres de las dimensiones que, según esta visión, componen el desarrollo: la salud (medida como esperanza de vida al nacer), la educación (la tasa de escolarización) y la riqueza (PBI per capita). La idea misma de desarrollo, entonces, admitía dimensiones distintas y complementarias y, por tanto, también la acción de las agencias internacionales debía modificarse para incluir sus (escasos) programas en el ámbito de la comunicación. Es bajo este impulso que, a partir de una iniciativa de la Organización para la Cooperación y el Desarrollo Económicos (OCDE) en 1996, toma fuerza la idea de acordar objetivos comunes en el marco de los organismos multilaterales para reforzar la cooperación internacional para el desarrollo. Los Objetivos del Milenio (ODM), lanzados en el año 2000 por la ONU, fueron el resultado de ese esfuerzo. Allí se condensaron ocho objetivos que todos los Estados adherentes deberían alcanzar antes de 2015, cada uno desglosado en metas y tiempos para su aplicación, y a su vez cada una de ellas acompañada de indicadores que ayudasen a la medición del cumplimiento.

De un rápido análisis de los objetivos, los indicadores y las metas de los ODM, puede interpretarse que la visión de la comunicación que los inspiró está fuertemente influenciada por el clima de época, ya que concentra su atención en el acceso a los recursos tecnológicos de la comunicación y no en la apropiación del proceso comunicacional. La principal referencia en este sentido se encuentra en la meta 8F: "en cooperación con el sector privado, dar acceso a los beneficios de las nuevas tecnologías, en particular los de las tecnologías de la información y de las comunicaciones". Y a esta meta le corresponden los indicadores 8.14 Líneas de teléfono por cada 100 habitantes, 8.15 Abonados a teléfonos celulares por cada 100 habitantes, y 8.16 Usuarios de Internet por cada 100 habitantes.

La agencia encargada de la recolección y análisis de estos indicadores es la Unión Internacional de Telecomunicaciones (ITU por sus siglas en inglés), organismo intergubernamental con una impronta profundamente tecnicista que en 2004 lanzó el Acuerdo para la Medición de las TIC para el Desarrollo, un ambicioso proyecto para medir el impacto de las 
TICs en los procesos de cooperación para el desarrollo ${ }^{18}$. Este proceso de tecnificación del rol de la comunicación en los debates sobre desarrollo se vio favorecido por la celebración de la Cumbre Mundial sobre la Sociedad de la Información (CMSI), iniciativa de la ITU, y se celebró en dos etapas: la primera en Ginebra en 2003 y la segunda en Túnez en 2005. Allí los representantes de los gobiernos adherentes, del sector privado y de la sociedad civil, siguiendo la línea trazada por el lanzamiento de los ODM ${ }^{19}$, elaboraron una declaración de principios y un plan de acción, concentrados en 10 puntos y sus líneas de intervención, además de la creación del Foro de la Gobernanza de Internet (Internet Governance Forum IGF) y el Grupo de las Naciones unidas sobre la Sociedad de Información (UN Group on the Information Society - UNGIS). Los 10 objetivos lanzados allí por la CMSI y aún hoy en evaluación fueron:

conectar a todos los pueblos a través de las TICs y generar puntos de acceso comunitarios; conectar a todas las escuelas primarias y secundarias a las TICs; conectar a todos los centros de investigación científica a través de las TICs; conectar todas las bibliotecas, museos, servicios postales y archivos nacionales a las TICs; conectar a todos los centros de salud y hospitales a las TICs; conectar a todas las dependencias gubernamentales y generar sitios web; adaptar todos los programas de las escuelas primarias y secundarias para enfrentar los retos de la sociedad de la información, teniendo en cuenta las circunstancias nacionales; asegurarse de que toda la población mundial tenga acceso a servicios de radio y televisión; alentar el desarrollo de contenidos y la puesta a punto de las condiciones técnicas para facilitar la presencia y uso de todos los idiomas del mundo en internet; asegurarse de que más de la mitad de los habitantes del mundo tengan acceso a las TICs a su alcance y hagan uso de ellas ${ }^{20}$ (ITU, 2014:2).

Estas líneas se convirtieron rápidamente en el plan de acción de las organizaciones multilaterales en las discusiones sobre desarrollo, inclusive en el ámbito post-2015, una vez cumplido el plazo establecido para el cumplimiento de los ODM. Las negociaciones para arribar a un consenso global sobre qué es el desarrollo y cuáles objetivos deben cumplir los países para llegar a él lograron la aprobación en el marco de las Naciones Unidas de la Agenda 2030 para el desarrollo sostenible, en agosto de 2015.

A riesgo de caer en cierto reduccionismo con respecto al amplio debate que precedió la aprobación de la agenda 2030, nos concentraremos aquí en comentar exclusivamente la visión de la comunicación que emana de los documentos oficiales al respecto. Una simple búsqueda a través de los 17 objetivos y 169 metas aprobadas nos da un primer -y triste-

18 CEPAL elaboró entre 2004 y 2014 informes sobre la aplicación de los sistemas de medición de la ITU en América Latina e inclusive elaboró propuestas para su adaptación a la realidad de los países no OCDE. Un completo análisis del impacto de las propuestas de los ODM en la región se puede encontrar en CEPAL (2011), Propuesta de indicadores complementarios para el monitoreo de los ODM: indicadores de acceso a las tecnologías de la información y las comunicaciones, Santiago de Chile: CEPAL

19 La "marcas de época" presentes en los documentos oficiales de las cumbres de Ginebra y Túnez son evidentes hasta en un primer análisis lingüístico. En ellos se hace especial hincapié en conceptos como necesidades, capacidades diferentes, entorno propicio, diversidad cultural, etc. El documento completo puede leerse en http://www.itu.int/net/wsis/docs/geneva/official/dop-es.html [último acceso 07/04/2019]

20 Traducción propia del inglés. 
panorama en cuanto al rol de la comunicación. La encontramos, en principio, en dos metas de implementación: una relativa al objetivo 5, el de lograr la igualdad entre los géneros y empoderar a todas las mujeres y las niñas ("5.b Mejorar el uso de la tecnología instrumental, en particular la tecnología de la información y las comunicaciones, para promover el empoderamiento de la mujer") y la otra en el objetivo 9, que se propone construir infraestructuras resilientes, promover la industrialización inclusiva y sostenible y fomentar la innovación ("9.c Aumentar de forma significativa el acceso a la tecnología de la información y las comunicaciones y esforzarse por facilitar el acceso universal y asequible a Internet en los países menos adelantados a más tardar en 2020"). Ambos puntos, 5 y 9 , fueron revisados en la cumbre de alto nivel realizada en 2017, y en ambos se ampliaron las metas para incluir la posesión y cobertura de teléfonos móviles (metas 5.B.1 y 9.C.1).

En ambos casos la comunicación es puramente instrumental, es decir que se la usa como instrumento para otro fin, se la asimila al uso de las TICS, y no a partir de la reflexión crítica sobre el proceso en sí mismo. Pero el lugar quizás de mayor protagonismo para la comunicación aparece en el objetivo 16: Promover sociedades pacíficas e inclusivas para el desarrollo sostenible, facilitar el acceso a la justicia para todos y crear instituciones eficaces, responsables e inclusivas a todos los niveles. La meta 16.10, "Garantizar el acceso público a la información y proteger las libertades fundamentales, de conformidad con las leyes nacionales y los acuerdos internacionales", es el único espacio de compromiso explícito suscrito con respecto a la comunicación en la agenda. Los indicadores de la meta 16.10 propuestos hasta ahora, sin embargo, profundizan su sentido e intención: 16.10.1 "número de casos verificados de homicidios, secuestros, desapariciones forzadas, detenciones arbitrarias y tortura de periodistas, personal asociado de los medios de comunicación, sindicalistas y defensores de derechos humanos en los doce meses previos"; y 16.10.2 "número de países que adoptan y aplican garantías constitucionales, estatutarias y/o políticas para el acceso público a la información"21.

Es decir, en un primer análisis del rol de la comunicación en los ODS de la Agenda 2030, podemos encontrar nuevamente los dos pilares que identificaban el paradigma difusionista de los años 50 y 60: el acceso a las tecnologías de la comunicación y la libre circulación de la información. Se trata de una visión claramente instrumental de la comunicación, que se completa con la incorporación de la lógica del mercadeo en la difusión de la propria agenda. De esta manera, se priorizó claramente la visión surgida de las CMSI (comunicación como instrumento) por sobre la de la WCCD (comunicación como un derecho).

Es verdad, de todas maneras, que los ODS no son la Agenda 2030 sino sólo una parte de ella. Si bien aún no se encuentren indicios acerca de la concepción participativa de la comunicación del desarrollo, los cinco pilares que mueven el espíritu de la Agenda 2030 personas, planeta, prosperidad, paz y alianzas- conforman un entorno favorable a priori para su instalación. Los primeros documentos elaborados acerca del objetivo 16 por ejemplo, reflejan una mayor transversalidad en su análisis. La IFLA, International Federation of Library Associations and Institutions, publicó un texto en el que advierte que para el cumplimiento del acceso a la información son necesarias cuatro claves: acceso a la infraestructura de comunicación e información, un contexto social positivo para su uso, capacidades suficientes en las comunidades y sus miembros, y un panorama legal favorable (IFLA, 2017:8). Si bien es verdad que estas propuestas abren una rendija para la elaboración de 
proyectos de comunicación participativa a partir de la cooperación internacional, su objetivo explícito (el acceso a la información) dista años luz de la praxis, la elaboración teórica y la experiencia de los medios comunitarios y las iniciativas horizontales de comunicación para el desarrollo.

\section{El posdesarrollo, la colonialidad y los últimos debates}

En los últimos años ha tomado fuerza, en las ciencias sociales latinoamericanas y de otras regiones tradicionalmente consideradas subdesarrolladas o en desarrollo, una vertiente teórica que cuestiona la idea de desarrollo desde sus fundamentos epistemológicos. El primer eje de esta crítica radical al conocimiento moderno deriva de su articulación intrínseca con la tradición y los axiomas cognoscitivos de la razón científica occidental, que anula o descarta cualquier conocimiento producto de cualquier "paradigma-otro". De allí que este grupo de autores hable de colonización del saber y del ser en las sociedades latinoamericanas (Barranquero-Carretero y Saez-Baeza, 2015:51). Existe entonces una geopolítica del conocimiento en la cual Occidente no sólo custodia el saber y la verdad sino también las vías para llegar a ella y las formas de enunciarla ${ }^{22}$.

El segundo gran eje de esta perspectiva está en el rechazo del concepto mismo de desarrollo en cuanto categoría cultural impuesta por el binomio modernidad/colonialidad. Se propone, entonces, la desconexión total de la categoría occidental y la construcción de un nuevo paradigma a través de bases epistemológicas que partan desde el Tercer Mundo e incluyan saberes y conocimientos descartados y ocultados por occidente. El Posdesarrollo surge así desde el rechazo a la idea misma de desarrollo fruto de la racionalidad científica occidental y el desonocimiento mismo del concepto de desarrollo como teoría/ideología impuesta colonialmente a los pueblos subdesarrollados (Cornago Prieto, 1997:81). En cambio, se propone un enfoque a partir de las epistemologías del sur, que rechaza la idea totalizante y universalista de la ciencia, se nutre de conceptos provenientes de la ecología, el paradigma del buen vivir, la idea de decrecimiento en función de las necesidades de la naturaleza, y una nueva razón que sea la "traducción" de experiencias concretas, cosmopolitas y vividas, por encima de la abstracción cientificista.

Esta perspectiva tuvo muy poco desarrollo en el ámbito de la comunicación, pero su aporte ayudó a plantear, por lo menos, dos interrogantes en torno a las prácticas de la comunicación para el desarrollo y para el cambio social. Cuestiona la raíz instrumental intrínseca a la concepción de comunicación para algo; esta idea condenaría la hiperespecializa-

22 Esta perspectiva, que en relaciones internacionales llamamos postcolonial, ha tenido fuertes desarrollos en todo el continente. Recomendamos la lectura de Quijano A. (2000), "El fantasma del desarrollo en América Latina", Revista venezolana de economía y ciencias sociales, Caracas, v.6 n², pp. 73-90, Quijano A. "Colonialidad del poder, eurocentrismo y América Latina", en Lander E. (comp. 2000) La colonialidad del saber: eurocentrismo y ciencias sociales. Perspectivas Latinoamericanas, Buenos Aires:CLACSO, pp. 201-246; un breve recorrido por los principales acervos y aportaciones teóricas de esta perspectiva se encuentran en Galindo Rodriguez F. (2013), “Enfoques postcoloniales en Relaciones Internacionales: un breve recorrido por sus debates y sus desarrollos teóricos”, Madrid, Relaciones Internacionales Revista académica cuatrimestral de publicación electrónica del Grupo de Estudios de Relaciones Internacionales (GERI), n22, pp. 85-107; y Boaventura de Sousa Santos (2007), Conocer desde el Sur. Para una cultura política emancipatoria, La Paz:CLACSO 
ción del campo y la imposibilidad de entrar en contacto con otras ciencias sociales y naturales. Al mismo tiempo, al tratarse de una perspectiva ligada a una cosmología-otra, que incluye a otros actores en su visión del mundo además del ser humano, también critica fuertemente el antropocentrismo del concepto de comunicación para el desarrollo o para el cambio social.

Muchas de estas concepciones provienen de los saberes y culturas originarias de América Latina que han sido fuente de un proceso de cambio muy importante en algunos países andinos. En la primera década del 2000, Venezuela, Ecuador y Bolivia modificaron sus textos constitucionales para incluir los conceptos quechua de sumak kawsay y aimara de suma qamaña. Traducidos rudimentariamente como "buen vivir" o "vivir bien", estos han modificado por completo la estructura del derecho público de esos países, subordinándolo en parte a cosmogonías no occidentales. La mayoría de estas concepciones entienden la comunicación como una fuerza transversal a todos los aspectos de la vida en el mundo y, por consiguiente, las leyes y las constituciones de esos países debieron ser adaptadas a estas ideas. Este factor, junto a la persistencia de un sector comunitario, alternativo y popular en el campo de la teoría y la práctica de la comunicación en América Latina, explica la aparición en el mismo periodo de leyes de regulación del espectro audiovisual, de la propiedad de los medios de comunicación o de su accionar en el espacio público en Venezuela (2005), Argentina (2009), Bolivia (2011), Ecuador (2013) y Uruguay (2014). En todos los casos es posible detectar continuidades con los debates que surgieron en los años 60, como la defensa de la soberanía cultural y la producción local; o novedades, como el reconocimiento oficial a formas participativas y cooperativas de comunicación. Estos procesos, aún en estudio y de difícil ejecución, pueden ser considerados también como nuevas fuentes de la perspectiva de la comunicación para el desarrollo en una apuesta que retoma la dicotomía PNC/Doctrina internacional. Cabe aclarar que la precedente oleada de reformas comunicacionales en el continente se realizó en el periodo de las dictaduras militares, la cual estuvo claramente influenciada por la doctrina del Free Flow of Information, como en el caso de los decretos regulatorios de la prensa o comunicación de Chile en 1982, Uruguay en 1977, Brasil en 1967, Ecuador en 1975 y Argentina en $1980^{23}$.

La actividad de los medios comunitarios en el mundo y sus redes son otra de las grandes fuentes de producción de prácticas y saberes que sigue alimentando el debate en torno a la comunicación. Desde la creación de medios como Indymedia, durante las protestas en Seattle contra la cumbre de la OMC en 1998, hasta en nacimiento de las TV comunitarias o TeleStreet y radio streaming en el seno de movimientos sociales y organizaciones barriales en América Latina, el dato de la apropiación de los recursos tecnológicos por parte de los sectores populares para la construcción de meta-discursos sobre comunicación y medios ha sido de lo más interesante y fructífero en el campo. Conceptos como contrahegemonía, antisistémico o praxis crítica, volvieron a tener resonancia en las teorizaciones sobre comunicación y desarrollo a pesar de contar con un lugar aún muy marginal.

23 Creemos aquí que la continuidad y evolución de estos paradigmas y sus agentes en nuestro continente no sin retrocesos o conflictos- los convierte en un conjunto dinámico que puja por la construcción de una identidad que a principio del siglo XXI intentó ponerse en juego en el complejo sistema internacional. Sería interesante en este sentido estudiar a las normas sobre comunicación latinoamericanas no tanto en su carácter jurídico, sino entendiendo según la perspectiva constructivista de las relaciones internaionales, que las normas son una de las formas que adopta lo ideacional, elemento fundamental del comportamiento de los estados en el sistema internacional. 


\section{Conclusión}

La comunicación para el desarrollo es un campo con una larga evolución histórica atravesado por tensiones y dinámicas propias de un campo del orden social de la humanidad, digno de estudios mucho más pormenorizados de los que hemos intentado realizar aquí. Su constitución se da en el marco del conflicto desatado entre los intereses de las principales potencias desarrolladas para incorporar el resto del mundo a su dinámica y visión del desarrollo, y la necesidad de la periferia del mundo de encontrar vías propias para la modificación de sus presentes. En esa tensión, que supera claramente el plano de lo económico y atraviesa las relaciones sociales, económicas políticas y hasta de la epistemología de las ciencias con la cual se analiza, también podemos afirmar que se encuentran representadas las diferentes formas de concebir la comunicación y su rol en el proceso.

Sin embargo, la evolución teórica de esa relación comunicación-desarrollo y los vaivenes en los debates académicos que en parte la definieron parecen haber superado largamente su puesta en práctica y asimilación en el orden internacional como factor indispensable para sostener cualquier política acerca del desarrollo. Los pilares comunicacionales que sostenían al paradigma de la modernización, la difusión de mensajes universales sin barreras -y, por tanto, la libre circulación de la información-y de las innovaciones tecnológicas en el ámbito de la comunicación persisten como principal preocupación de las instituciones internacionales dedicadas al desarrollo a pesar de que ya se haya demostrado su perjudicialidad para las comunidades y los países subdesarrollados. El paradigma participativo vio en la idea de comunicación para el cambio social su último gran intento de convertirse en herramienta transversal para el desarrollo, despojado de su visión crítica acerca de la estructura de poder, dominación y dependencia que sostiene la cooperación internacional. He aquí el primer gran desafío del campo: destecnologizar su percepción y entendimiento en los ámbitos de la cooperación internacional al desarrollo. Por otro lado, y si bien el NOMIC y el Informe MacBride representaron una derrota en este sentido, queda aún pendiente la superación de la visión que reduce la comunicación al acceso a la información, para comenzar a ponerla en práctica como derecho a comunicar de todos los seres humanos, en cuanto productores y distribuidores de mensajes.

Desde esa realidad, y de la práctica de las políticas relativas al desarrollo a nivel internacional, se desprenden otros retos para el campo; entre ellos, el de redescubrir su origen cuestionador y crítico de la realidad social. Una comunicación constreñida dentro del marco de las instituciones internacionales es una comunicación amputada de su potencial más preciado e importante, que es su lado transformador. Pero ¿quiénes donarían para proyectos de Comunicación para el Cambio Social, si el cambio social significara cuestionar y combatir los privilegios de los donantes? La influencia de los donantes y del sistema internacional de cooperación es patente también en otras dinámicas de la comunicación para el desarrollo. La necesidad, por ejemplo, de medir resultados a través de metas, indicadores y estadísticas cuantificables plantea otro gran desafío para los enfoques participativos: ¿Cómo medir la apropiación de tecnologías y medios de comunicación sin caer en la cuantificación numérica de aparatos y accesos? ¿Cómo cuantificar el grado de participación y el aporte cultural originario en la comunicación?

Es necesario entonces que el campo se abra a las perspectivas nuevas que han proliferado en la práctica, las leyes y la academia, y que pueda recuperar el impulso perdido tras la década de los 80 para volver a levantar algunas de esas banderas hoy renovadas. Hace 
falta abrirse al cuestionamiento de las perspectivas epistemológicas clásicas, recuperar el motor de la praxis, codo a codo con los actores que están planteando nuevas formas de entender la comunicación y el desarrollo para poder renovar los marcos teóricos de su estudio. Quizás, aún frente a un proceso en marcha, como el de la Agenda 2030, no sea todavía demasiado tarde para aportar enfoques y visiones que ayuden a ajustar en función participativa la evaluación del proceso.

\section{Bibliografía}

Arenal Del C. (1985), "El nuevo orden mundial de la información y de la comunicación", en: Revista de Estudios Internacionales, Madrid, v. 6, n¹, pp. 7-39.

Banco Mundial (2007), World congress of communication development. Lessons, Challenges, and the Way Forward, Washington: The World Bank.

Baran P. A. y E. J. Hosbawm, (1963), "Las etapas del crecimiento económico", en: México, El Trimestre Económico, v. 30, no118, pp. 284-295.

Barbero J. M. (1987), De los medios a las mediaciones. Comunicación, cultura y hegemonía. BarceIona: Gustavo Gili.

Barranquero-Carretero A. y C. Sáez-Baeza, (2015), "La crítica descolonial y ecológica a la comunicación para el desarrollo y el cambio social”, Madrid, Palabra Clave, n. 18, pp. 41-82.

(2010), “Comunicación alternativa y comunicación para el Cambio social democrático: Sujetos y objetos invisibles en la enseñanza de las Teorías de la comunicación". Congreso internacional AE-IC Malaga "Comunicación y desarrollo en la era digital". Universidad Autónoma de Barcelona.

Beltrán R. (2006), "La comunicación para el desarrollo en América Latina: un recuento de medio siglo”, en: Anagramas Rumbos y Sentidos de la Comunicación, Medellin, v. 4, no8, pp. 53-76

Castro Ruano J. L. (1999), "Medios de comunicación y relaciones internacionales", en: Cursos de Derecho Internacional de Vitoria-Gasteiz, Universidad del País Vasco, pp. 175-233

Cimadevilla G. (2008), "Cinco tesis y una semblanza. Trayectos académicos en la convergencia comunicación-desarrollo", en: Revista Latinoamericana de Ciencias de la Comunicación, Brasilia, no 8-9, pp. 100-110.

Cornago Prieto N. (1997), “Desarrollo, subdesarrollo y postdesarrollo: un análisis crítico del debate contemporáneo", en: Cursos de Derecho Internacional de Vitoria-Gasteiz, Universidad del País Vasco, no1, pp. 39-88.

FAO (2007), Comunicación y desarrollo sostenible, Roma: FAO.

FAO (2008), Manual diagnóstico participativo de la comunicación rural, Roma: FAO.

Fox de Cadorna E. (1978), "Políticas Nacionales de Comunicación”, en: Revista Chasqui, Quito, no 21, pp. 91-97.

Gray-Felder D. y J. Deane, (1999), Comunicación para el Cambio Social. Documento programático e informe sobre una conferencia, New York: Rockefeller Foundation.

Gumucio Dargon A. (2001), Haciendo olas. Historias de comunicación participativa para el cambio social, New York:Rockefeller Foundation. 
Gumucio Dargon A. (2010), "Cuando el doctor no sabe. Comentarios críticos sobre promoción de la salud, comunicación y participación", en: Estudios sobre las Culturas Contemporáneas, Colima, v. 16, no 31, pp. 67-93.

International Federation of Library Associations and Institutions IFLA (2017), Development and Access to Information 2017, Holanda: IFLA.

International Telecommunication Union ITU (2014), Final WSIS Targets Review - Achievements, challenges and the way forward, Ginebra: ITU.

Mattelart A. (1996), La comunicación mundo. Historia de las ideas y de las estrategias, México D.F.: Siglo XXI.

Mattelart A. y M. Mattelart (1997), Historia de las teorías de la comunicación, Barcelona: Paidós.

MacBride S. (1980), Un solo mundo, voces múltiples, Ginebra: UNESCO.

McAnany E. (2010), “Communication for development and social change: New Millenium”, en: Communication research trends, Santa Clara, no3, vv. 29, pp. 3-43.

Mefalopulos, P. (2008), Development communication sourcebook : broadening the boundaries of communication. Washington DC: World Bank.

Quirós F. y Sierra F. (Editores 2016), El espíritu MacBride. Neocolonialismo, comunicación-mundo y alternativas democráticas, Quito: CIESPAL.

Roncagliolo R. (1989), “Comunicaciones y desarrollo en América Latina”, Madrid, Telos, no17 pp. 80-87.

Sen A. (2001), "Las teorías del desarrollo en el siglo XXI", Madrid, Leviatan, no 84, pp. 65-84.

Schiller E (1976), Comunicación de masas e imperialismo yanqui, Barcelona:Gustavo Gili.

Servaes J. (1999), Communication for development: one world multiple cultures, New Jersey: Hampton Press.

Tufte T. y P. Mefalopulos, (2009) Participatory communication: a practical guide. Washington, DC: World Bank.

Unicef (2011), Comunicación para el desarrollo. Fortaleciendo la eficacia de las Naciones Unidas, New York: ONU. 